\title{
LONG-WAVELENGTH GERMANIUM PHOTODETECTORS BY ION IMPLANTATION
}

I.C. Wu*, J.W. Beeman, P.N. Luke, W.L. Hansen, and E.E. Haller*

*Dept. of Materials Science

University of California at Berkeley

and

Engineering Division

Lawrence Berkeley Laboratory

1 Cyclotron Road

Berkeley, CA 94720

Presented at the Fall 1990 Meeting of the Materials Research Society Boston, Nov. 26-Dec. 1, 1990.

This work was supported by NASA Contract No. W-14606 under Interagency Agreement with the Director's Office of Energy Research, Office of Health and Environmental Research, U.S. Department of Energy under Contract Numbers DE-AO376 SF00098 and DE-AC03-76SF00098. 
I.C. Wu(1,2), J.W. Beeman(1), P.N. Luke(1), W.L. Hansen(1), and E.E. Haller(1.2) Lawrence Berkeley Laboratory(1) and Department of Materials Science and Mineral Engineering(2) ,University of California, Berkeley, CA 94720, USA

\section{ABSTRACT}

Extrinsic far-infrared photoconductivity in thin high-purity germanium wafers implanted with multiple-energy boron ions has been investigated. Initial s'sults from Fourier transform spectrometer(FTS) measurements have demonst ated that photodetectors fabricated from this material have an extended longwavelength threshold near $192 \mu \mathrm{m}$. Due to the high-purity substrate, the ability to block the hopping conduction in the implanted IR-active layer yields dark currents of less than 100 electrons/sec at temperatures below $1.3 \mathrm{~K}$ under an operating bias of up to $70 \mathrm{mV}$. Optimum peak responsivity and noise equivalent power(NEP) for these sensitive detectors are $0.9 \mathrm{~A} / \mathrm{W}$ and $5 \times 10^{-16} \mathrm{~W} / \mathrm{Hz}^{1 / 2}=1$ $99 \mu \mathrm{m}$, respectively. The dependence of the performance of devices on $t$.. residual donor concentration in the implanted layer will be discussed.

\section{NTRODUCTION}

Because of the potential technological importance of high performance far infrared derectors for space-born telescope applications, the development of far infrared Ge detectors using two-layer structure based on the Blocked. Impurity-Band(BIB) concept is being extensively studied 1-5. In contrast to normal photoconductors 1,3 , BlB photodetectors have number of desirable features: 1. extension of the cutoff wavelength limit from the dopant photoconductive onset to longer wavelengths, 2. a decrease in interference from cosmic radiation because of volume reduction, and 3. a reduction in the noise due to fixed unity gain.

These improvements have been realized to a large extent with $\mathrm{Si} B I B$ detectors for wavelengths up to $30 \mu \mathrm{m}^{6.7}$. Furthermore, these novel devices have the capability of achieving background limited performance at the very low infrared levels encountered in space-based observations8. In addition, twodimensional monolithic BIB detector arrays have been fabricated using IC process technologies 6.7 .

Conventional BIB detectors consist of a thin, undope-t intrinsic blocking layer grown homo-epitaxially on an intermediately doped substrate. Incident photons absorbed in this intermediately doped region generate free carriers which pass through the pure layer, and are collecied as photocurrent when the device is properly biased. The ionized impurity state travels via successive bound cartiers to the opposite electrode. The intrinsic layer serves to block the large currents which would arise from hopping conduction within the impurity band in the intermediately doped layer. The device derives improved performance from the fact that the presence of the blocking layers makes it possible to dope the IR-active layer more heavily than in conventional photoconductive detectors. without the corresponding increase in dark current 
due to hopping conduction. The higher doping also allows a reduction in device volume without compromising quantum efficiency of the device.

In view of considerable success of Si BIB detectors, it appears worthwhile to try to develope Ge detectors with similar structure. Because of the much smaller ionization energies of shallow impurities in $\mathrm{Ge}$ as compared with $\mathrm{Si}$, Ge BIB detectors are expected to have a longer wavelength cutoff around $200 \mu \mathrm{m}$ and perhaps beyond. $\mathrm{A}$ Ge BIB detector of conventional design consists of a pure Ge epitaxial layer which is grown on an intermediately doped IR-active substrate. Early devices fabricated by chemical vapor deposition (CVD) ${ }^{9}$ indeed showed an extended long-wavelength threshold at $190 \mu \mathrm{m}$ and had a promising peak responsivity of $5 \mathrm{~A} / \mathrm{W}$. However, data on Ge BIB devices which exhibit sucb characteristics as well as low dark currents $(<100 \mathrm{e} / \mathrm{s})$ and NEP10.11 which. are necessary for future satellite bom applications have not been published so far.

In this paper, we present a new approach of fabricating two layer Ge farIR photoconductors which show BIB device characteristics. In this new approach, the IR active layer is formed in an ultra-pure Ge substrate using ion implantation. The high-purity substrate serves as the blocking layer. Since the substrate can be selected from very high quality bulk crystals( $I \mathrm{Na}_{\mathbf{a}}-\mathrm{N}_{d} \mid<2 \times 10^{10}$ $\left.\mathrm{cm}^{-3}\right)^{12.13}$, its ability to block hopping conduction is assured. The thickness and dopant concentration of the IR-active layer can be controlled by varying ion energies and doses. Since complete analysis of BIB device physics and applications have been published elsewhere1-5, we will confine ourselves to describing the processes required to make the new structure and to their characterization. Preliminary experimental results of the discrete far-IR Ge detectors will be presented and discussed.

\section{EXPERIMENTAL DETAIS}

The starting material for these devices investigated in the present work was prepared from ultra-pure Ge with extremely low impurity concentration (about $2 \times 10^{10} \mathrm{~cm}^{-3}$ ) and essentially perfect crystalline quality13. Wafers of 200 $\mu \mathrm{m}$ thickness were cut from large boules with a diamond saw, then lapped and polish etched to remove all mechanical damage. Boron ions were implanted using several energies to approximate at impurity concentration profile of $3 x$. $1016 \mathrm{~cm}^{-3}$ from the surface to depth of $0.5 \mathrm{\mu m}$. The wafers were then annealed for one hour at $400^{\circ} \mathrm{C}$ in argon to remove any implantation damage and to fully activate the boron 14. Boron was used for implantation doping because it is the shallowest elemental acceptor in $G e$ and it is active as implanted at room temperature. These two characteristics guarantee the longest wavelength response and circumvent very high temperature annealing which might introduce other impurities into the wafer. Furthermore, boron is the lowest mass acceptor thus reaching the greatest implantation depth for a given ion energy.

A schematic diagram of a prototype ion implantation Ge BIB device described in this work is demonstrated in Fig. 1 . Square samples of $1 \times 1$ or $2 \times 2$ $\mathrm{m} \mathrm{m}^{2}$ were cut from an implanted and annealed wafer with a dicing saw. The small samples were mounted with the implanted side down onto a glass slide using wax. The device was then further thinned by chemically etching the unimplanted side to reach a final sample thickness of SO $\mu \mathrm{m}$. The sides were 


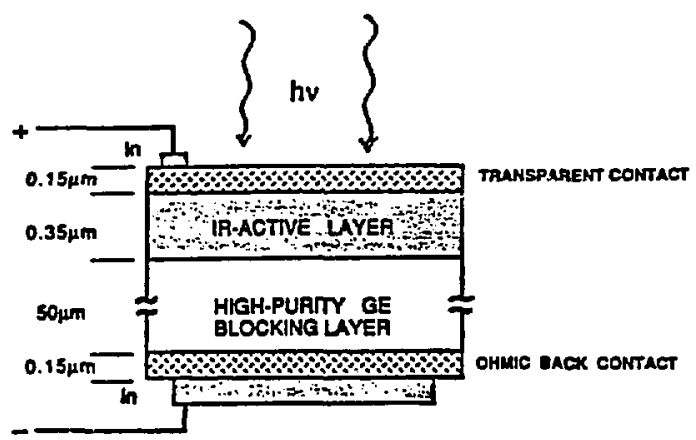

XC. $1010-3359$

Fig. 1 Schematic cross section of 2 ion implanted Ge far-IR detector.

also etched to remove any residual saw damage, which could contribute to excess surface leakage currents. A final implantation of $\mathrm{B}^{+}$ions formed a degenerately doped $p^{++}$contact on the top face without any post-implant annealing. We chose an implant dose of $2 \times 10^{13} \mathrm{~B}^{+} \mathrm{cm}^{-2}$ at an energy of $25 \mathrm{keV}$. This produce ohmic, semi-transparent contact layers. 15

Finally, a strip of copper was attached to the bottom surface of the detector using pure indium solder. The specimens were then mounted on copper heatsinks in an integrating cavity for photocurrent response and NEP tests. A copper wire was pressure-welded to the top surface by using a pure indium pad. Measurements of dark current were obtained using an integrating JFET amplifier JF-4 (Infrared Labs.) which has a Iead noise of less than 20 electrons. 16

\section{EXPERIMENTAL RESULTS AND DISCUSSIONS}

Various detector figures of merit, including spectral response, Iesponsivity, noise equivalent power, and dark current, were measured from several specimen. Because all the devices showed very uniform response, we chose to restrict ourselves to reporting results from just one of these specimen. The two spectral responses of this detector under different bias are shown in Fig.2. It was obtained using a Fourier transform spectrometer (FTS). The periodic variation in the current response resulted from the Fabry-Perot interference between the front and the back surfaces of the detector. When the applied bias was varied from 10 to $25 \mathrm{mV}$ with the detector operating at a temperature of 1.5K, the long-wavelength cutoff moved from 140 to $192 \mu \mathrm{m}$, significantly beyond the $120 \mu \mathrm{m}$ onset of a standard boron-doped Ge photoconductors 17 . Such a shift does not occur in the very pure blocking layer. It must be due to photoexcitation in the moderately doped ion-implanted layer.

BIB device structure can be compared to a metal-insulator-semiconductor (M1S) photodiode. A positive bias applied to the detector surface contact (Fig. 1) will deplete the moderately doped detection layer of ionized acceptors to some depth. The positively charged minority donors constitute the space charge. By solving Poission's equation, we find that the width of depletion layer $W$ relates to the applied bias $V_{2}$ and minority donor concentration $N_{d}$ as follows:

$$
w=\sqrt{\frac{2 \varepsilon_{0} \varepsilon V_{2}}{e N_{d}}+t^{2}}-t
$$


where $E$ is the dielectric constant of Ge, the thickness of blocking layer, and $e$ the electronic charge. It is desirable to increase the applied bias to widen of the depletion layer $W$ from the tail of the implant profile into the intermediately doped plateats so as to extend the cutoffs to the longest possible wavelengths and also to increase the quantum efficiency of the detector. However, for our ion implanted BIB detectors, no further increase in both cutoff wavelength and photoresponse were observed when the bias voltage exceeded $25 \mathrm{mV}$. Beyond $25 \mathrm{mV}$, the detector noise level increased sharply with the increase of the applied bias.

It should be mentioned that photocurrent could be also measured under reverse bias operation. This response has been found to be due to the implanted contact on the back side of the device as shown in Fig.1. However, the spectral response (Fig.2) generated from the back single-implant contact is different from that the front multiple-implant side. The former always lacks a sharp long wavelength threshold and shows a lower value of the peak responsivity at about $60 \mu \mathrm{m}$. For a conventional extrinsic photoconductor, the spectral response is completely symmetric with respect to applied bias polarity. However, for these implanted detectors, the asymmetry of performance in regard to bias polarity is a strong indication that the spectral response (Fig.2) of the detector comes primarily from the front multiple-implant layer.

In Fig.3, we show three figures of merit of this device. The absolute responsivity and NEP values at $99 \mu \mathrm{m}$ as a function of bias were tested at a photon background of $1 \times 10^{8}$ photons $/ \mathrm{cm}^{2}$-s and at a typical photon chopping frequency of $23 \mathrm{~Hz}$ with a low noise, trans-impedance preamplifier and a spectrum analyzer. Dark currents at this test temperature of $1.3 \mathrm{~K}$ were found to be independent of the bias up to $40 \mathrm{mV}$. Operated at $24 \mathrm{mV}$, the detector has a NEP of $5 \times 10^{-16} \mathrm{~W} / \mathrm{Hz}^{1 / 2}$ which is only about an order of magnitude higher than the value of background limited performance (BLIP). Scaled to the measured responsivity at $99 \mu \mathrm{m}$, the corresponding peak responsivity in Fig.2 is expected to be about $0.9 \mathrm{ANW}$ at $120 \mathrm{\mu m}$.

The thickness of the detection layer for these devices is determined by two parameters, $N_{d}$ and $t$. For instance, with an applied bias of $25 \mathrm{mV}$, a value of $t=50 \mu \mathrm{m}$, and an assumed $N_{d}=1 \times 10^{11} \mathrm{~cm}^{-3}$, the depletion width $W$ has a value of

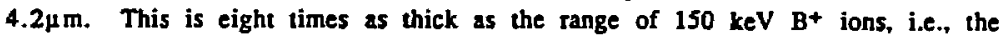
device is fully depleted except the electrode layer. But, for $N_{d}=1 \times 10^{12} \mathrm{~cm}^{-3}$, with
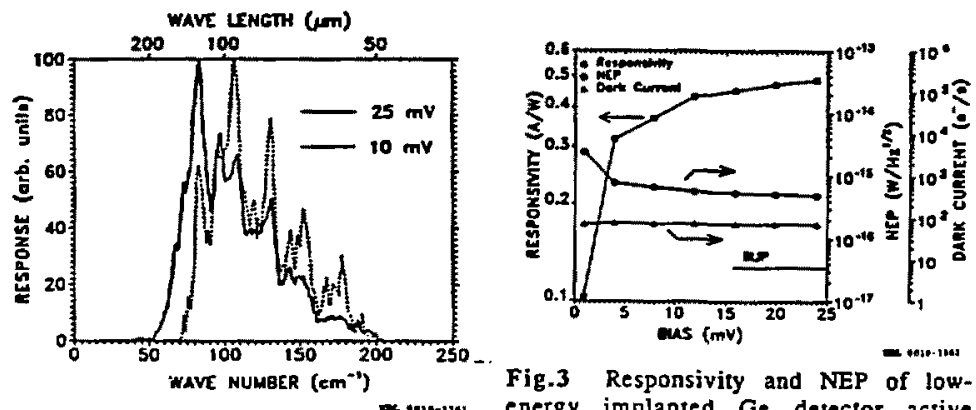

Fig.3 Responsivity and NEP of lowenergy implanted $G e$ detector active

Fig.2 Spectral tesponse of a boronimplanted $G e$ detector under two differen: bias 10 and $25 \mathrm{mV}$. layer depth $=0.5 \mu \mathrm{m}_{*}[\mathrm{~B}]=3 \times 1016 / \mathrm{cm}^{3}$. narrow band fiter at $99 \mu \mathrm{m}$, chopping frequency $=23 \mathrm{~Hz}$. 
all other parameters as above, $W$ would be $0.44 \mu \mathrm{m}$ which is close to depth distribution of $150 \mathrm{keV} \mathrm{B}^{+}$ions. Therefore, the determination of $\mathrm{N}_{d}$ in the IRactive layer is crucial for device development. Due to the fact that carrier freeze-out does not occur in the intermediately doped layer, both the actual value of $N_{d}$ and therefore $W$ in the implanted IR-active layer are not known and are still under investigation. We have used variable temperature Hall effect measurements to determine donor concentrations on several low dose Bimplanted layers $\left([B]=1 \times 10^{15} \mathrm{~cm}^{-3}\right)$. These low dose layers were deliberately utilized to avoid hopping conductivity in the implanted layer at low temperatures. Our preliminary Hall data indicate that $150 \mathrm{keV}$ boron asimplanted layers are highly compensated with donor concentrations in the $1 \times 10^{14} \mathrm{~cm}^{-3}$ range. Post-implant annealing steps for these implanted layers have been found to be necessary to lower donor concentrations to the $1 \times 1013$ $\mathrm{cm}^{-3}$ range, which is still higher than the expected value of $\mathrm{Nd}_{d}<1 \times 10^{12} \mathrm{~cm}^{-3}$. As already mentioned, the lower donor concentration values lead to an increased depletion region thickness, therefore, if the Iesidual donor concentration $\mathrm{N}_{\mathrm{d}}$ can be reduced to lower values ( $N_{d}-1 \times 10^{11} \mathrm{~cm}^{-3}$ ) via optimization of the fabrication processes, then the use of $\mathrm{MeV}$ implantation to produce IR-active layer several microns in thickness to improve the defector sensitivity become very desirable.

Finally, low dark current is a key factor which promises the detector performance in low background condition. The far-IR space-based astronomy application requirements for dark current are less than $100^{\circ}$ electrons/sec/pixel with a typical pixel size of less than $0.5 \times 0.5 \mathrm{~mm}^{2}$. As shown in Figs.3 and 4 , even lower dark currents, about $70 \mathrm{e} / \mathrm{s}$, have been achieved with this discrete Ge device $1 \times 1 \mathrm{~mm}^{2}$ in size at operating temperatures below $1.3 \mathrm{~K}$ and a bias below $70 \mathrm{mV}$. The temperature dependence of dark currents under different bias conditions is shown in Fig.4. Indeed, at a temperature of $1.25 \mathrm{~K}$, the value of the dark current did not exceed $100 \mathrm{e} / \mathrm{s}$ up to an applied bias of $80 \mathrm{mV}$. The breakdown voltage in the dark for this operating temperature was about $90 \mathrm{mV}$.

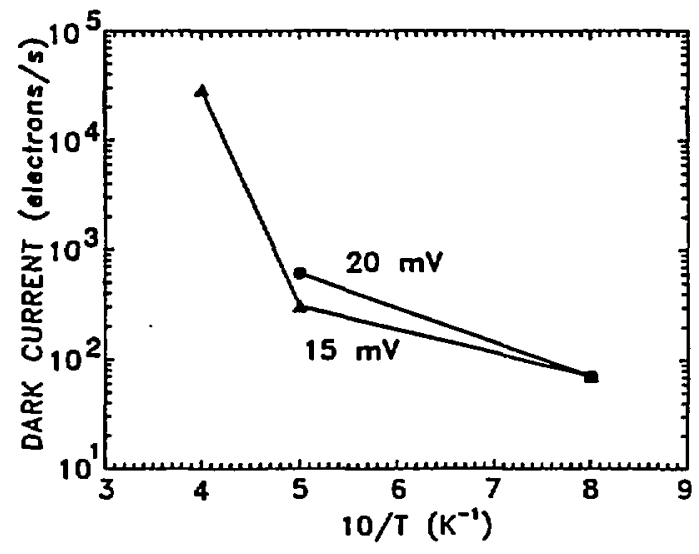

xec $1010-3060$

Fig.4 Dark currents as a function of temperature. At $1.25 \mathrm{~K}$, the values of dask currents are less than $70 \mathrm{e} / \mathrm{s}$ over the applied voltages from 1 to $70 \mathrm{mV}$. 
CONCLUSION

We have fabricated the first far-infrared Ge detectors based on the BIB concept using ion implantation technique. These boron-implanted Ge BIB detectors exhibit operating characteristics compatible with requirements for low background applications. Device parameters such as low dark currents, acceptable sensiti;ity and extended wavelength threshold, demonstrate that ion-implanted Ge far-IR detectors offer promise for use in astrophysics instrumention. Further studies are aimed at fully understanding the device operation. In particular, the origin of the residual donor concentration in the ion-implanted IR-active layer needs to be determined. If the lower $N_{d}$ value can be achieved in boron implanted layers, the use of high-voltage ion implantation to incretase the active layer thickness will also be examined in order to optimize the detector performance.

This work was supported by NASA Contract No. W-14606 under Interagency Agreement with the Director's Office of Energy Research, Office of Health and Environmental Research, U.S. Department of Energy under Contracl Numbers DE-A03-76SF00098 and DE-AC03-76SF00S15.

\section{REFERENCES}

1 See articles in " Proc. 3rd IR Det. Techn. Workshop, NASA Tech. Memo 102209", C. MeCreight, ed., (1989).

2. M.D.Petroff and M.G. Stapelbroek, U.S. Patent No. 4-568-960(4 February 1986).

3. V. Hadek, J. Farhoomand, C.A. Beichman, D.M.Watson and M.D. Jack, Appl Phys. Letl. 46, 403(1985).

4. F. Szmulowicz and F. Madar, J. Appl. Phys. 62,2533(1987).

5. M.G. Stapelbroek, M.D. Petroff, J.J. Speer, D.D. Arington, and C. Sayre, IRIS Specialty Group in IR Detectors, Boulder, CO, August 4, 1983.

6. D.B. Reynolds, D.H. Seib, S.B. Statson, T. Herter, N. Rowlands, and J Schoenwald, IEEE Trans, NS 30, 857(1989).

7. T. Herter, N.Rowlands, S.Y.W. Beckewith, and G.E. Gull, "Proc. 3rd IR Det Techn. Workshop, NASA Tech. Memo 102209", C. McCreight, ed., 427(1989).

8. E.E. Haller, M. R. Hueschen, and P. L. Richards, Appl. Phys. Lett. 34,495(1979).

9. D.M. Watson and J.E. Huffman, Appl. Phys. Lett 52 1602(I988).

10. C.S. Rossington, Ph.D. dissertation, University of California, Berkeley, 1988.

11. I.C.Wu (unpublished work).

12. E.E. Haller, W.L.Hansen, and F.S. Goulding, Adv. in Plıys. 30, No.1, 93(1981).

13. W.L.Hansen and E.E. Haller, Mat. Res. Soc. Symp. Proc. 16, I(1983).

14. K.S. Jones and E.E. Haller, J. Appl. Phys. 61, 2469(1987).

15. Y.Hadek, D.M. Watson, C.A. Beichman, and M.D. Jack, Phys. Rev. B 31, 630(1985).

16. Infrared Labs. Inc. Product Brochure, April, 1989, p.30.

17. H. Shenker, W. J. Moore, and E. M. Swiggard, J. Appl. Phys., 35, 2965(1964). 